\title{
An Axiomatic Approach to Human Behavior ${ }^{\mathrm{i}}$
}

\author{
Sheldon Grant Levy \\ Wayne State University, USA
}

Copyright $(2017$ by authors, all rights reserved. Authors agree that this article remains permanently open access under the terms of the Creative Commons Attribution License 4.0 International License

\begin{abstract}
Since Euclid's development, the axiomatic method has appealed to a limited number of non-mathematicians. These include Newton's laws of motion, Spinoza's The Ethics, and the political philosophy represented in the United States Declaration of Independence. In psychology, the notable efforts have been those of Hull and more recently of Smedslund. However, none of these examples have stated the premises in symbolic logic notation. An illustrative set of postulates for human behavior is presented in which only the relationships---"not", "and", "or" and "implies"--- are employed. These propositions include basic concepts such as anxiety, aggression, and identification and fundamental empirical results, for example, the basic law of effect of Thorndike. The model then capitalizes on Muncaster's PropCalc which provides a computer analysis of a set of propositions expressed in symbolic notation for their consistency and redundancy. The procedure also provides a basic set of deductions implied by the propositions. Examples of conclusions derived from the model are presented and limitations of the prototypical model are discussed. The focus of the paper is on illustrating the utility of the axiomatic approach for theory development in psychology.
\end{abstract}

Keywords Psychological Theory, Axiomatic Method, The PropCalc, Symbolic Representation, Theoretical Constructs

\section{Introduction}

Curiosity is a basic human motive and there have been several models developed by psychologists utilizing this concept (Silva [1]). The motive is also present in other species and represents an attempt to understand. Understanding reduces anxiety that arises from uncertainty in combination with a perceived threat. The understanding informs behavior. When there is anxiety, that behavior attempts to achieve and maintain reduced anxiety. ${ }^{1}$ The

1 This approach differs from that of Kruglanski [3] who posited that knowledge-seeking was a result of a more specific purpose or goal. Curiosity here is posited as a general motivation to know to reduce uncertainty and that uncertainty arises from a wide variety, almost pervasive, set of unknowns. curiosity motive may also involve inquisitiveness about an observation that may be unique to the individual. However, even that inquisitiveness, once activated, seeks to reduce uncertainty or ambiguity although it may not involve a threat. The curiosity motive may also involve inquisitiveness about an observation that may be unique to the individual. Explanation is a cognitive representation of understanding and consists of the ability to inform the self and, for some, others about the process that resulted in understanding. Not all understanding requires explanation. Particularly for humans, there are expressive or emotional means of understanding as represented in art, music, dance and drama, Eisner [4], although Eisner specifically includes experience rather than expressiveness as a basis for understanding. The difference is between understanding how to paint well and the painting itself as an expressive statement of understanding or meaning. Expressiveness may also at tunes be simply an overt representation of internal feelings. When understanding occurs, it may not be veridical nor is it mandatory that it be conscious.

\section{Background}

One approach to explanation was that of Euclid (c. 325-265 B.C.E./Fitzpatrick [5]) through his axiomatic method. This form of explanation involved several components. First, there were the concepts such as point and line. Even when not fully defined, they included attributes. Thus, a point was a dimensionless location and a line was unidimensional. Some precision in concepts was necessary for unambiguous communication, a necessity for explanation. Secondly were the axioms. These were basic assumptions. They were not reducible to more fundamental components. The axioms have also been characterized as "self-evident", Ross [6].). For example, the first axiom of Euclid states that if two separate entities are equal and an equal amount is added to each, they remain equal. Euclid also included postulates in the premises, for example, that a line can be drawn from one point to another. The third component consists of the rules by which one classifies and relates the elements to each other. Aristotle (384-322 B.C.E.) preceded Euclid and established the basis for formalization, ultimately in symbolic form (Aristotle, c: 350, [7]). In this paper, the development is based on four fundamental relationships--not (A or not $\mathrm{A})$, and $(\mathrm{A}$ and $\mathrm{B})$, or $(\mathrm{A}$ or $\mathrm{B})$ and implies (A 
implies B). A fourth aspect of analysis was a reliance on a reasoning process. Thus it is not possible for an entity to be both itself and not itself ( $\mathrm{A}$ and not $\mathrm{A}$ ) at the same time. This is the basis of an alibi in legal proceedings. If one can convincingly present evidence that the accused was somewhere other than the scene of the crime, then the defense may argue that the defendant could not have been in both places at the same time. The deductive process led to both the discovery and the explanation of many relationships. Euclid was able to derive, during his lifetime, 465 conclusions from his set of axioms, postulates, and definitions and the allowed reasoning process (Wilder [8], p. 4).

As a formal system of reasoning, however, the "explanations" of the Euclidean system were of abstractions. It, however, was possible to relate the abstract system to the real world through a correspondence between the elements of the system and those of external reality, and thus the design of the building was improved through application of the Euclidean deductions.

Insight is one variation of understanding, but the level of conscious awareness that is necessary is unclear. It is apparent that animals below man, some far below (such as flying birds) are capable of insight-problem solving (Heinrich \& Burgnar [9], Taylor [10], for dogs, PBS [11] and for donkeys, see Huffington Post [12]). Problem solving in organisms less cognitively developed than humans appears to involve the basic logical relationships of--"not", "or", "and", and "implies". The examples illustrated by the ravens/crows and by the dog's successful search for the stuffed doll "Darwin" provide evidence for the possibility that the system of reasoning is not structured on pure whimsy but derives from some fundamental biological understanding. (Intrinsic logic for humans was considered by Barwise and Etchemendy [13], p. 1). This does not prevent humans from developing whimsical systems.

\subsection{Historical Applications of the Axiomatic Method}

The assumptions that are incorporated as starting principles in a deductive system will include all of the following terms: assumptions, premises, axioms, and postulates. Derivations are labeled as theorems or conclusions or deductions. The assumptions are not necessarily "self-evident" or a "common notion", but appear to be a fundamental principle, not easily reducible to components, from which a large number of deductions might be derived. Newton (1643-1727) labeled his laws of motion as "axioms" (Suppes [14]). On rare occasions the axiomatic method appealed to philosophers. Spinoza, (1632-1677) attempted to develop an axiomatic system of ethics based on fundamental propositions (Spinoza, [15]). Each of the parts of his treatise begins with definitions, axioms, and propositions. The first four of the seven axioms in the first section "Concerning God" provide insight into his classic treatise. They involve basic relationships such as (A or B) (Axiom I) and strong deterministic (cause and effect) assumptions (Axioms III and IV).
I. Everything which exists, exists either in itself or in something else.

II. That which cannot be conceived through anything else must be conceived through itself.

III. From a given definite cause an effect necessarily follows; and, on the other hand, if no definite cause be granted, it is impossible that an effect can follow.

IV. The knowledge of an effect depends on and involves the knowledge of a cause.

In 1776, the American revolutionaries wrote a declaration of independence that appears to represent an attempt to develop a political philosophy based on deductions from fundamental principles (Pullano [16], Klau [17]):

"We hold these truths to be self-evident, that all men are created equal, that they are endowed by their Creator with certain unalienable Rights, that among these are Life, Liberty, and the pursuit of Happiness - That to secure these rights, Governments are instituted among Men, deriving their just powers from the consent of the governed,- That whenever any Form of Government becomes destructive of these ends, it is the Right of the People to alter or to abolish it, and to institute new Government, laying its foundation on such principles and organizing its powers in such form, as to them shall seem most likely to effect their Safety and Happiness.",2

\subsection{The Axiomatic Method in Psychology}

There have been few attempts in psychology to capitalize on the axiomatic method as a systematic approach to explaining human behavior. Hull attempted to accomplish this. His first postulate, which is really a composite of many assumptions, illustrates some of the difficulties in his approach. (Hull, [19], p.47.)

"Postulate 1

When a stimulus energy (S) impinges on a suitable receptor organ, an afferent neural impulse (s) is generated and is propagated along connected fibrous branches of nerve cells in the general direction of the effector organs, via the brain. During the continued action of the stimulus energy (S), this afferent impulse (s), after a short latency, rises quickly to a maximum of intensity, following which it gradually falls to a relatively low value as a simple decay function of the maximum. After the termination of the action of the stimulus energy (S) on the receptor, the afferent impulse (s) continues its activity in the central nervous tissue for some seconds, gradually diminishing to zero as a simple decay function of its value at the time the stimulus energy (S) ceases to act."

Abelson and Rosenberg [20], presented a mathematical approach to psycho-logic to account for "irrationality" in

2 The Declaration was formally analyzed in Levy [18] 
attitudes. The mathematical representation in social psychology has occurred periodically and mathematical models in psychology have had a venerable history. However, the specific form represented in the axiomatic approach has been substantially absent. More recently, Smedslund [21] has argued for the importance of the axiomatic method and provides an example in his health model. Amgoud and De Saint-Cyr [22] have presented a sophisticated mathematical approach to the axiomatic method and applied it to persuasive dialog. They conclude that the inconsistencies in arguments between two sides presenting themselves to a public (Holland-Sarkozy presidential election in France) lead to the conclusion that persuasion and reasoning must be considered as different processes.

However, neither Hull nor Smedslund capitalized on symbolic logic. Neither did Spinoza or the authors of the Declaration of Independence but symbolic logic did not develop until approximately the mid $-19^{\text {th }}$ century. Even had that development occurred earlier, it is unlikely that a symbolic logic representation would have stirred citizens to revolution. Words in language have an important emotional component as well and provide subtleties difficult to include in a formal model (Barwise and Etchemendy [13], p. 4, Zajonc [23]). Although Newton's laws of motion were also delineated earlier, he avoided the limitation by developing the calculus (a system also credited to Leibnitz but apparently formally conceived much earlier by Archimedes, 287-212 B.C.E. (Rehmeyer [24]).

\subsection{Formalization of Axioms}

Even with the efficiencies of symbolic logic, the procedures for both developing and evaluating a set of assumptions, or model, as well as the process of deriving conclusions, has frequently been time-consuming. It is possible to restate the symbolic relationships in a form amenable to computerized analysis and as a result quickly assess the integrity of the model (the logical consistency of the premises), the presence of redundancies, and to obtain a number of conclusions. The Muncaster PropCalc (Muncater, [25]) provides a means by which this may be accomplished. The PropCalc is briefly described and then applied to a prototypical model of human behavior.

\subsection{Basic Symbolic Representation}

Four relationships are fundament to the development and analysis that is presented. These are, and, or, not, and implies. They are symbolized respectively as $\wedge(\mathrm{A} \wedge \mathrm{B}), \vee(\mathrm{A} \vee \mathrm{B})$, - $(-\mathrm{A})$, and $\Rightarrow(\mathrm{A} \Rightarrow \mathrm{B})$. Statements may be converted to symbols and related to each other in the assumptions by one of the four relationships. Thus, if $\mathrm{A}=$ "All men are created equal" and B = "Governments derive their power from the consent of the governed", the symbolic representation for both would then simply be $(\mathrm{A} \wedge \mathrm{B})$. Once the translation has been completed, the basic rules of deduction may be applied to derive conclusions. One such rule, for example, is Modus Ponens. It states that if $\mathrm{A}$ is true and if $\mathrm{A}$ implies $\mathrm{B}(\mathrm{A} \Rightarrow \mathrm{B})$, then B is true. Thus, if an assumption (premise) is A (as defined above from the Declaration of Independence), and a second premise is that $(\mathrm{A} \Rightarrow \mathrm{B})$ where $\mathrm{B}$ was defined previously, then Modus Ponens states that one may deduce that B is true. In this example, A implies B is an assumption. That assumption may be an incorrect statement about reality. In this case, conclusions derived from the model would not be supported by scientific evidence. The verbal descriptive approach would be unwieldy if applied to a large set of premises that might be necessary for a theory of human behavior. The symbolic notation is:
1. A
(Assumption 1 (or postulate or premise)
2. $(\mathrm{A} \Rightarrow \mathrm{B})$ (Assumption 2)
3. B (Conclusion applying Modus Ponens)

\subsection{The PropCalc}

The ProCalc is a computerized aid to the analysis of the logic of verbal statements (Zinnes [26]) and may be considered analogous to a statistical program for numerical analysis. The PropCalc provides automated analyses once the symbolic representations have been appropriately reformulated. In addition to evaluating consistency, and redundancy, an additional procedure, labeled simplification, allows the selection of a conclusion and a determination of the assumptions that were required to reach that conclusion. The PropCal also lists, for each conclusion derived from the model, the steps of the reasoning process through which that conclusion was obtained' Zinnes [27]. has provided a prominent example of the value of analyzing extant social science theory through formalization and PropCalc analysis.

Since the set of deductions does not exhaust the creativity of the human mind, it is possible that an important conclusion for which the assumptions were designed does not appear in the list of conclusions. To determine whether that conclusion was derivable from the model, it is possible to include it among the postulates. A redundancy analysis would establish whether the conclusion was derivable from the other principles. However, if it were, the redundancy analysis would not automatically provide the reasoning sequence by which that conclusion may be obtained.

\subsection{An Illustrative Axiomatic Approach to Human Behavior}

A general model of human behavior requires a systems approach. First, it is necessary to formulate postulates of individual behavior that include basic concepts as well as empirical generalizations derived from well-established research such as the law of effect (Brown \& Hernnstein [28]), conformity (Asch [29], Allen and Levine [30]), obedience (Milgram [31], prejudice and stereotypes (Devine [32]) or Stevens' power law (Brown \& Herrnstein [29]). In addition, important variables and behaviors, such as inter-group conflict, the effects of propaganda, and decision-making in the legal system, eventually require representation, either through postulates or, preferably, as deductions from a basic set of premises. This requires the development of local-models for specific areas in which concepts and 
postulates are then incorporated into the general model (Levy [33]). The development of a model would therefore be iterative.

There are at least two major problems in attempting to initially develop an all-encompassing model. The first is that the set of postulates would probably be unnecessarily large. A major goal is efficiency. That goal is more likely attained through an initial basic set. The second and related problem is that there are many aspects of human behavior that may not be salient during the initial formulation. An iterative process is more likely to lead to an efficient increase in comprehensiveness.

\subsection{The Initial Model}

A set of concepts were first defined and then the postulates were formulated in both verbal and symbolic form. Finally, the model was subjected to PropCalc analysis. Sample conclusions are presented after conversion to their verbal equivalent.

\subsection{Concepts}

The following were defined: symmetry, anxiety, dominance, threat, aggression, hatred and arousal. The symbols are in parens. The focus in this model was on human conflict.

Symmetry (Sy): Symmetry and imitation are not identical. Symmetry represents an internal response that is similar to that which would occur if the observer were actually engaged in the action being observed. There is physiological support for symmetry from mirror neurons (Molenberghs, Cunnington, and Mattingley [34]). It does not require the conditions specified by Bandura, Ross and Ross [35] for imitation. Imitation is a transformation of the internal reaction to overt behavior. Identification is also closely related to imitation but not identical. The fan in the stadium who excitedly exclaims at the end of an American football game, "We Won!," presents an example of identification.

Arousal (ar): A heightened mental and physiological alertness and awareness.

Anxiety (Ax): A negative state of arousal that arises from threat combined with uncertainty.

Dominance (D): Dominance requires a hierarchical group organization. The dominant organism has fewer restrictions on behavior and the ability to restrict the behavior of those lower in the hierarchy (Brown [36] Brown \& Herrnstein [28]).

Threat (T): Threat arises from a perceived risk. The risk is most often to physical well-being but may also be to one's self-image or general well-being including economic security.

Aggression (A): Any destructive act and is, therefore defined by behavior. This definition does not require that the act be against a con-specific nor does it require the determination of the intent of the actor.
Hatred (H): Hatred is hostility combined with denigration of the target. Hatred may be a distinctly human quality although less intense emotions may exist in other species, notably simians. It requires symbolic processes and a memory. Hostility involves the mobilization of aggressive impulses and is an internal state. Hatred provides a symbolic dominance through the denigration of the target. It is almost always directed toward another human (or con-specific).

The hypothesis that links symbolic processes to overt activity has not been developed in this paper. It is apparent that hatred is more likely to lead to overt aggres0sion toward the denigrated target which is frequently dehumanized. Hatred may generalize to a group sharing characteristics with a target and may be expressed in mass murder/rape/torture. The reverse is also possible. Hatred of a group generalizes to individuals who share salient characteristics. Since hatred achieves, through symbolic means, dominance, it may be self-rewarding and increase the probability of future hatred. ${ }^{3}$ This process was summarized in a remark by Eric Hoffer, "Hate is cheap." 4

\section{Postulates and Discussion}

\section{Sy}

2. Sy $\wedge($ Ig $) \Rightarrow$ Id).

Symmetry implies an in-group (Ig) and identification (Id) with the in-group. Identification with the in-group is required to avoid a symmetry response to predators. Among less complex organisms, this is probably an automatic biological process. For humans, us-them distinctions are heavily dependent on socialization.

\section{3. $(-\operatorname{Ig} \wedge T) \Rightarrow A x$}

An out-group (-Ig) and threat implies anxiety (Ax), (a threatening out-group implies anxiety).

4. $\mathbf{A x} \Rightarrow-\mathbf{A x b}$ : Anxiety implies behavior to reduce the anxiety $(-A x b)$.

5. $(A x \wedge D) \Rightarrow-A x$

Dominance implies a reduction in anxiety (-Ax). As a logical symbol, -Ax represents a reduction in anxiety including its absence.

\section{6. $(\mathrm{Ag} \wedge \mathrm{D}) \Rightarrow$ Agf}

Aggression that leads to dominance which is, by postulate 5 , anxiety reducing, is more likely to occur in the future (Agf). If aggression is self-rewarding, then $\mathrm{Ag}$ implies $\mathrm{Agf}$ but this relationship has not been included.

\section{7. $($ Ag $\wedge$-D) $\Rightarrow$-Agf}

8. H $\Rightarrow$ Hf: Hatred implies future hatred'

9. Nar $\Rightarrow$ Hg: Negative arousal leads to high generalization

3 An early behaviorist explanation of repression was based on the law of effect. To apply the law, a thought was considered a response and the affect associated with it an outcome. Thoughts that resulted in negative affect, for example, memories of traumatic events, were not likely to occur in the future.

4 Hoffer was a commissioner on the Commission on the Causes and Prevention of Violence. The remark at a meeting of the commissioners in 1968. Unfortunately, there is no record of the remark except the memory of the author. 


\section{Par $\Rightarrow-\mathrm{Hg}$ :}

Positive arousal leads to lower generalization than negative arousal. Here, -Hg represents a level lower than that of $\mathrm{Hg}$.

The ten postulates are presented in Table 1.

Table 1. Postulates of the illustrative model

\begin{tabular}{|ll|}
\hline 1. & Sy \\
3. & $(-\mathrm{Ig} \wedge \mathrm{Ig} \wedge \mathrm{T}) \Rightarrow \mathrm{Id})$ \\
4. & $\mathrm{Ax} \Rightarrow-\mathrm{Axb}$ \\
5. & $(\mathrm{Ax} \wedge \mathrm{D}) \Rightarrow-\mathrm{Ax}$ \\
6. & $(\mathrm{Ag} \wedge \mathrm{D}) \Rightarrow \mathrm{Agf}$ \\
7. & $(\mathrm{Ag} \wedge-\mathrm{D}) \Rightarrow-\mathrm{Agf}$ \\
8. & $\mathrm{H} \Rightarrow \mathrm{Hf}$ \\
9. & $\mathrm{Nar} \Rightarrow \mathrm{Hg}$ \\
10. & $\mathrm{Par} \Rightarrow-\mathrm{Hg}$ \\
\hline
\end{tabular}

\section{Analysis}

The PropCalc analysis determined that the postulates did not contain either contradictions or redundancies. The following deductions, translated to their verbal equivalents, illustrate the advantages of the program as a basis for further development. The logical steps by which the deduction was obtained were provided by the analysis.

Conclusion 17: If there is either the absence of an outgroup or a lack of threat, there will not be anxiety. (Obtained from DeMorgan's theorem and postulate three.)

Conclusion 24: A lack of future aggression implies that there is not both aggression and dominance. (Transposition and postulate 6.)

Conclusion 36: If there is either an outgroup or a lack of identification, there will not be symmetry. (DeMorgan's theorem and the first conclusion after the postulates (fact 11).

\section{Summary and Discussion}

The axiomatic method has been demonstrated to constitute a powerful analytic and deductive form. Its application has appealed to both scientists as well as philosophers. However, although occasionally discussed, there have been few formal attempts in psychology to formulate models on an axiomatic basis and symbolic representations are very rare.

\subsection{The Value of the PropCalc}

The Consistency of a Psychological Theory

Verbal expression is the predominant form of psychological theory and it is from these statements that predictions to empirical investigations are derived. The complexity of a series of verbal statements may result in a set that contains contradictions. If contradictions are not present, then the model may be appropriate as a basis for deductions that imply expected outcomes. For this process to attain legitimacy, therefore, a) the verbal statements must be logically consistent, and b) the predictions must be logically derivable from the premises. By stating the verbal description in symbolic form, the PropCalc provides a means of assessing both. As discussed, the absence of a conclusion does not automatically determine whether it is capable of being derived from the model but it is also possible that the theorem does not logically follow from the premises. This evaluation of psychological theory has obvious merit. Levy [18] has illustrated the process in evaluating Devine's theory of stereotypes (Devine [32]. The exemplary publication is that of Zinnes [27] who subjected the theory of the democratic peace (two states with democratic governments are less likely to be engaged in armed conflict with each other than other combinations of political systems) and demonstrated that the theory was consistent and the primary conclusion was derivable from the model. The analysis was not a validation of the theory but established a critical requirement, that it possesses logical integrity.

\subsection{Assessing the Necessity of a Hypothetical Construct}

Psychologists frequently rely upon hypothetical constructs (MacCorquodale \& Meehl, [37]). Constructs are defined here simply as unseen element in a theory that provide greater understanding of and predictability to behavior than might be possible in their absence. The model represented in Table 1 included a number of these. In fact, only aggression and dominance were defined by behavior. Constructs are not inappropriate in theoretical formulations. They have been common in natural science. For example, the Greek Atomists explained everything that exists as consisting of atoms (Lucretius [38]). Early formulations in science, such as magnetism, might also be considered theoretical constructs. These examples represent a category that may be labeled "hypothesized entities". That is, although the recourse is to unseen elements, there is a belief, followed by appropriate research, that their existence may be established. Psychology also employs constructs, and sometimes these are also hypothesized entities. Thus, learning is unseen but inferred from behavior and may be considered a hypothesized entity because the physiological correlates that represent memory are expected to exist and will eventually be identified. Nevertheless, psychology incorporates a large number of unseen elements that are models or "as-if" constructs. The premier example may be Freud's concepts of the id and the ego (Freud [39]) and subsequently the superego. Freud explained that one should not expect to uncover mental structures that represent these constructs. They were a model, an "as-if" set of premises that were intended to explain some puzzling aspects of behavior. The PropCalc does not assist in categorizing constructs. However, it provides a means by which the necessity of the construct may be evaluated. This may be accomplished through the procedure of simplification. Some conclusions are central to the goals of the theory. The process of simplification determines which premises are required to obtain that conclusion. If the identified premises do not include constructs, then it is obvious that the construct was superfluous to a central focus of the model. In the postulates in Table 1, there are only two behaviorally defined terms, aggression and dominance. Only 
postulates 6 and 7 do not contain constructs. Of the three conclusions presented for illustration, only the second, (\#24) was obtained without recourse to constructs.

\section{Limitations of the Present Model}

\subsection{Similarity among Concepts}

Symmetry rather than imitation has been defined as a fundamental concept. Both imitation and identification may be derivable from symmetry but that remains for future development. Similarly aggression, anger, hostility and hatred are related. The first has been defined by behavior. Only hatred has been separately defined but it is an internal state as are anger and hostility. These may represent variations in intensity on a dimension of antagonism so that all the concepts are not required directly in the assumptions.

\subsection{Definitions}

The model builder has the freedom of including undefined terms and of providing definitions that are somewhat arbitrary. However, if a model is to be relevant to reality, a correspondence between a concept and a real counterpart is required. A number of concepts that appear to be common in psychology have been incorporated. These include: anxiety, aggression, threat, hatred, arousal, and identification. Others are of more common usage such as threat, although a psychological definition was incorporated in the premises. Still others are common in psychology but may be considered cross-disciplinary such as dominance, and in-group and out-group. An examination of definitions provided by the American Psychological Association in its glossary does not provide sufficient justification for adopting those definitions (APA, [40]). For most of the concepts the glossary did not provide any definition and for the exceptions, the definitions were imprecise. Aggression provides an example (italics added): "Aggression. Behaviors that cause psychological or physical harm to another individual." Among the difficulties with this definition are: a) it requires acts against a con-specific ("another individual"), b) surgeons are aggressive because they cause physical harm, (but their actions are not destructive) and c) it includes psychological harm. Thus, an act cannot be classified without knowledge of the reaction of the target. If a woman were told that she was overweight, the remark was aggressive if she were chagrined, but if the same remark were considered comedic, perhaps because she had just been named Miss Universe, then the comment was not aggressive.

At least as questionable was the definition of anxiety (italics added): "Anxiety. An intense emotional response caused by the preconscious recognition that a repressed conflict is about to emerge into consciousness." This clinical definition has several difficulties including, a) the emotional response is not identified as negative, $b$ ) as defined, anxiety requires an understanding of pre-consciousness and repression and, c) it contains conditions that are more limited than those of Freud [39]. Anxiety was a core concept of Freud's theory. The complexity of the concept is represented in the lectures for example pages 267- 272 and $489-513$ ). Freud recognized anxiety from external events: "Realistic anxiety is a reaction to the perception of danger"(p. 489). Realistic anxiety was distinguished from neurotic anxiety, "...the generation of anxiety is the ego's reaction to danger and the signal for taking flight"(p. 503). When a forbidden wish is threatening, there may be distortion, but anxiety may take "...the place of distortion of wish-fulfillment in anxiety dreams, ...generally for strong wishes" (p. 270). Anxiety has been defined as a general concept in the model but would not be inconsistent with Freud's discussion of either realistic or neurotic anxiety.

\subsection{Further Development, the Group and its Coordination}

Although the model included some fundamental assumptions related to human behavior, the logic that governs the integration of the individual into the group and the coordination of the group members in which there is both conformity and obedience, as well as the important explanation of conflict between con-specific groups, has not yet been developed. As stated at the outset, the primary purpose of this analysis has been to present the value of the axiomatic approach to theory development in conjunction with the PropCalc through the presentation of an illustrative rather than definitive model.

\section{REFERENCES}

[1] Levy, S. G. (2015b) An Axiomatic Approach to Human Behavior. Psychonomic Society, 56 ${ }^{\text {th }}$ Annual Meeting, November 21, Chicago.

[2] Silva, P. J. (2014). Curiosity and motivation. Ch. 10, 157-166. In Ryan, R. M. (Ed.) The Oxford Handbook of Human Motivation. New York: Oxford: Oxford University.

[3] Kruglanski, A.W. (1980) Epistemo-Logic-Process and Contents: Another Look at Attribution Theory. Psychological Review, 87(1), 70-87.

[4] Eisner, E.W. (1993). Forms of understanding and the future of educational research, Educational Researcher, 22, (7) 5-11. Access at:

http://www.jstor.org/stable/1176749?seq=1\#page_scan_tab_ contents

[5] Euclid (circa 300 B.C.E/2008.). Elements. Fitzpatrick, R., translation and edited. The elements of Euclid's geometry. ISBN 978-0-6151-7984-1. Retrieved from:

http://www.google.com/url?sa=t\&rct=j\&q=\&esrc=s\&source =web\&cd=1\&ved=0ahUKEwiL2omA37LRAhVM6oMKHS i5DQkQFggcMAA\&url=http $\% 3 \mathrm{~A} \% 2 \mathrm{~F} \% 2 \mathrm{Ffarside}$.ph.utexas .edu $\% 2$ FBooks $\% 2 F E u c l i d \% 2 F E l e m e n t s . p d f \& u s g=A F Q j C N$ Fek8wsmce-Szo2IA8hidHr1vopmw

[6] Ross, K.L. (2016). Euclid's axioms and postulates. Retrieved from: http://www.friesian.com/space.htm

[7] Aristotle (circa 350 .B.C.E./2017) Organon and other works. (W.D. Ross, editor). Retrieved from: https://archive.org/stre am/AristotleOrganon/AristotleOrganoncollectedWorks_djvu .txt 
[8] Wilder, R. L. (1965). Introduction to the foundations of mathematics. New York: John Wiley and Sons

[9] Heinrich, B. and T. Bugnyar (2007). Just how smart are ravens? Scientific American, April, pp. 64-71.

[10] Taylor, A. (2014). Are crows the ultimate problem-solvers?-Insider the animal mind. BBC 2, broadcast January 30, posted, February 4 at: https://www.youtube.com/watch?v=AVaITA7eBZE

[11] PBS (2011). How Smart are Dogs? Nova science now. Posted February 9, 2011 at:

http://www.pbs.org/wgbh/nova/nature/how-smart-dogs.html Transcript at: http://www.pbs.org/wgbh/nova/nature/how-smart-dogs.html

[12] Huffington Post (2017). Smart Donkeys use awesome problem-solving. Posted January 07. URL: http://www.huffi ngtonpost.com/entry/clever-donkey-crosses-fence-italy-vide o_us_5870a027e4b099cdb0fd6279 Video at https://www.youtube.com/watch?v=w0aER6cRCl0

[13] Barwise J. and J. Etchemendy. (1992.) The language of first order logic. Stanford: Center for the study of language and information.

[14] Suppes, P. (1974). The axiomatic method in the empirical sciences. Proceedings of the Tarski Symposium, Proceedings of Symposia in Pure Mathematics. 25, 465-479. Providence, RI: American Mathematical Society.

[15] Spinoza, B. (1675) The ethics. Translated from the Latin by Translated from the Latin by R. H. M. Elwes. Posting Date: May 28, 2009 [Ebook \#3800] project Gutenberg e-book] at: http://www.gutenberg.org/cache/epub/3800/pg3800.txt

[16] Pullano, F. (2012) The axiomatic method. Retrieved from: faculty.winthrop.edu/pullanof/.../The\%20Axiomatic\%20Met hod.pdf

[17] Klau, D. (2017). The Declaration of Independence as Euclidian Geometry. Retrieved from:

https://appealinglybrief.com/2015/12/09/the-declaration-of-i ndependence-as-euclidean-geometry/

[18] Levy, S.G. (2015a). The Role of Logic in Testing and Developing Psychological Theory: The Value of Some Formality, Division 24, APA, Society for Theoretical and Philosophical Psychology. March 5, Salt Lake City.

[19] Hull, C. L. (1943). Principles of Behavior. New York: Appleton-Century-Croft

[20] Abelson, R.P. and Rosenberg, M. J. (1958.) Symbolic psycho-logic: A model of attitudinal cognition. Behavioral science, 3, p. 1-13.

[21] Smedslund, G. (2000). A Pragmatic basis for judging models and theories in health psychology: The axiomatic method. Journal of Health Psychology, 5(2) 133-149.

[22] Amgoud, L. \& De Saint-Cyr (2013. An Axiomatic Approach for Persuasion Dialogs. Proceedings of the 2013 IEEE 25th International Conference on Tools with Artificial Intelligence, ICTA, Nov. 4-6. Washington, D. C. P. 618-625.

[23] Zajonc, R. B., (1980). Feeling and Thinking: Preferences Need No Inferences. American Psychologist, 35, 151-175.

[24] Regneyerm H, (2007). A prayer for Archimedes. Science News, posted Oct 3. at: url: https://www.sciencenews.org/article/prayer-archimedes

[25] Muncaster. R. G., (2017) http://www.math.uiuc.edu/ muncast/Applets/Applets34E72 A/index.html

[26] Zinnes, D. A. (2005.) Territoriality, contiguity and international conflict. Paper presented at the annual meeting of the International Studies Association, Hilton Hawaiian Village, Honolulu, Hawaii accessed at: $\mathrm{http} / / /$ citation.allacademic.com/meta/p_mla_apa_research_ci tation/0/7/2/0/5/pages72055/p72055-1.php

[27] Zinnes, D. A. (2004.) Constructing Political Logic: The Democratic Peace Puzzle. Journal of Conflict. Resolution, 48 (3) 430-454.

[28] Brown, R \& Herrnstein, R.J. (1977) Psychology. New York: Little, Brown.

[29] Asch, S.E. (1955). Opinions and social pressure. Scientific American, 193, 31-35.

[30] Allen, V. L., \& Levine, J. M. (1968). Social support, dissent and conformity. Sociometry, 138-149.

[31] Milgram, S. (1974) Obedience to authority: an experimental view. New York: Harper and Row

[32] Devine, P. (1989) Stereotypes: Stereotypes and prejudice: Their automatic and controlled components. Journal of Personality and Social Psychology, Vol. 56(1), Jan 1989, 5-18.

[33] Levy (2014) From human aggression to group conflict: An axiomatic approach. Conflict Research Society, University of Leeds, September 1-4, 2014. Paper posted as: An axiomatic approach to human aggression and group conflict: Model building from the outside-in.

http://www.crsconference.net/papers/sheldon_levy_axiomati c approach.pdf

[34] Molenberghs P, Cunnington R, Mattingley J (2009). Is the mirror neuron system involved in imitation? A short review and meta-analysis. Neuroscience \& Biobehavioral Reviews 33 (1): 975-980.

[35] Bandura, A., Ross, D., and Ross, S.A. (1961). Transmission of aggression through imitation of aggressive models. The Journal of Abnormal and Social Psychology, 63(3), 575-582.

[36] Brown, R (1965). Social Psychology. New York: Free Press.

[37] Maccorquodale, K. and Meehl, P. E. (1948). On a distinction between hypothetical constructs and intervening variables. Psychological Review, 55, 95-107.

[38] Lucreius, (c. 50 B.C.E./2016). On the nature of things. Translated by William Ellery Leonard. Download at: http://classics.mit.edu/Carus/nature things.mb.txt

[39] Freud, S. (1917/1977). Introductory lectures on psycho-analysis. Strachey, J. (Translator). New York: W. W. Norton-Liverright

[40] APA (2017). Glossary of psychological terms. From Gerrig, Richard J. \& Philip G. Zimbardo. (2002). Psychology And Life, 16/e. Published by Boston, MA.: Allyn and Bacon. Accessed at:

http://www.apa.org/research/action/glossary.aspx 